\title{
Breakdown of proteoglycan and collagen induced in pig articular cartilage in organ culture
}

\author{
J. T. DINGLE, P. HORSFIELD, HONOR B. FELL, AND M. E. J. BARRATT* \\ From the Tissue Physiology Department, Strangeways Research Laboratory, Cambridge, and the \\ Immunology Division, Department of Pathology, University of Cambridge
}

\begin{abstract}
Dingle, J. T., Horsfield, P., Fell, H. B., and Barratt, M. E. J. (1975). Annals of the Rheumatic Diseases, 34, 303-311. Breakdown of proteoglycan and collagen induced in pig articular cartilage in organ culture. Explants of articular cartilage from young pigs were maintained in organ culture for 10-16 days, and degradation of matrix was induced by retinol or complement-sufficient antiserum. The percentage breakdown of proteoglycan and collagen (as hydroxyproline release) was measured. The response of the cartilage depended on whether or not the explants were cut so as to include some of the invading marrow ('invasion zone'). In media containing retinol, cartilage lost up to three-quarters of its proteoglycan whether the invasion zone was present or not, but very little of its collagen unless this region was included. In the presence of complement-sufficient antiserum, however, cartilage without the invasion zone was virtually unaffected, but both proteoglycan and hydroxyproline were released when invasion zone was included; here proteoglycan release began almost immediately, but there was a time-lag of 6-8 days before a substantial amount of hydroxyproline appeared in the medium. Histological examination of sample explants from the experiments supported the biochemical findings. The possible significance of the results in relation to rheumatoid arthritis is discussed.
\end{abstract}

The mechanical, chemical, and biological properties of articular cartilage largely depend upon the molecular structure of the extracellular matrix, and loss of one or both of the two major polymers of matrix, i.e. proteoglycan or collagen, results in the drastic changes in properties observed in pathological conditions such as rheumatoid arthritis. To determine the enzymatic mechanisms of the disease process it is important to ascertain the sequence of events in cartilage erosion, and in particular the relative susceptibility of collagen and proteoglycan to degradation in the living tissue. In this investigation an attempt has been made to determine the relative rates of catabolism of these two molecules in resorbing articular cartilage.

In previous work experiments were made to investigate the histological effects of excess of retinol (Barratt, 1973) and of complement-sufficient rabbit antiserum against pig erythrocytes (Fell and Barratt, 1973) on pig articular cartilage in organ culture. It was found that medium containing either added retinol or antiserum and complement had little (retinol) or virtually no (antiserum and complement) effect on cartilage devoid of soft connective tissue, but caused severe depletion, as indicated by loss of metachromasia, when the cartilage was associated with either invading marrow or an adjacent explant of synovial and capsular tissue.

It was decided to analyse some of the biochemical processes underlying these morphological changes. In the present experiments, explants of articular cartilage, with or without invading marrow, were cultured in medium containing heat-inactivated normal rabbit serum (NRS), NRS with added retinol (R + NRS) or rabbit antiserum to pig erythrocytes plus rabbit serum complement $\left(\mathrm{AS}+\mathrm{C}^{\prime}\right)$. The rates of release of proteoglycan and collagen under these different conditions were studied.

\section{Material and methods}

ORGAN CULTURE

Tissue

Cartilage was sliced from the articular surfaces of the 3rd 


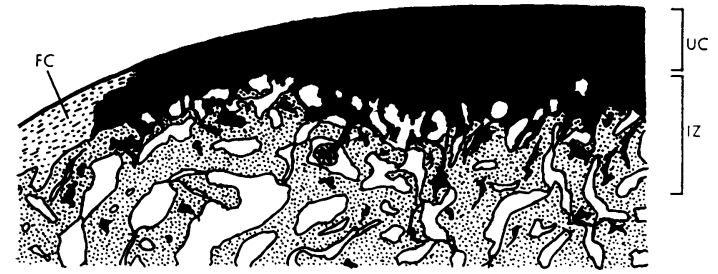

FIG. 1 Diagram of articular cartilage and subchondral bone. Black = cartilage; stippled = bone; clear $=$ invading marrow; $U C=$ uncalcified cartilage; $I Z=$ incasion zone; $F C=$ fibrous transitional cartilage (not used)

and 4th metacarpals of young pigs aged 22-32 weeks; except for one experiment the animals were considerably older than those used in previous work (Fell and Barratt, 1973; Poole, Barratt, and Fell, 1973; Barratt, 1973) and weighed approximately $110-160 \mathrm{~kg}(250-350 \mathrm{lb})$ as against $55-90 \mathrm{~kg}(125-200 \mathrm{lb})$ in the earlier experiments.

Two types of explant were made (Fig. 1). One consisted of isolated cartilage; in the other the cartilage was cut at a deeper level, so as to include the blind ends of the cavities excavated by the invading marrow in the calcified region ('invasion zone') and sometimes also some of the underlying trabecular bone. The explants were carefully paired, one of each pair receiving the experimental treatment and the other serving as its control. To prepare explants of isolated cartilage a long, thin strip was cut from each condylar ridge of the metacarpals and divided into two approximately equal parts about 4-5 $\mathrm{mm}$ in length. Comparable explants of the second type, i.e. cartilage with the invasion zone, were obtained by excising about 8 fragments from the condyles, and from these selecting 2 pairs, the members of each pair being similar in size and in the amount of invasion zone present.

\section{Culture method}

The organ culture technique was the same as that previously described by Fell and Barratt (1973) except that in all but one of the present experiments the explants were laid directly on a stainless steel grid in the culture vessels instead of on a piece of millipore filter supported by the grid as in the earlier work. As before, the medium was changed every two days and at each change the used medium from each culture dish was collected separately for biochemical examination.

\section{Media}

The basic medium was the chemically defined solution $\mathrm{BGJ}_{5}$ (Fell and Dingle, 1969). Retinol (Roche Products) in ethanolic solution was added to a medium composed of $10 \%$ normal heat-inactivated rabbit serum (NRS) in $\mathbf{B G J}_{5}$, or, in one experiment (Fig. 3), normal heatinactivated pig serum (NPS) at a concentration of 3.3 $\mu \mathrm{g} / \mathrm{ml}$ of the final medium. The same amount of ethanol only was added to the control medium (10\% NRS in $\mathrm{BGJ}_{5}$ ).

Rabbit antiserum (AS) to pig erythrocytes was prepared as follows. Well washed $(\times 6)$ pig erythrocytes of either group $\mathrm{A}$ or group $\mathrm{O}$ were injected intraperitoneally into rabbits. Each course consisted of 3-4 daily injections of $1-10 \%$ erythrocytes suspended in saline; there were resting intervals between courses of 2-8 months. The pooled antisera used in the experiments reported here $\stackrel{\mathbb{D}}{\stackrel{D}{ }}$ were of the 5th course with an antiglobulin titre of $1: 40003$ and a haemolytic titre of $1: 400$. Fresh, unheated rabbit $\square$. serum was used as a source of complement $\left(\mathrm{C}^{\prime}\right)$ (see Fell $\stackrel{\text { ? }}{\text { ? }}$ and Barratt, 1973). For the final medium $5 \% \mathrm{AS}+10 \% \overrightarrow{\mathrm{F}}$ $\mathrm{C}^{\prime}$ was added to $\mathrm{BGJ}_{5}$. Control medium consisted of $\stackrel{\text { ? }}{\frac{9}{9}}$ pooled $15 \% \mathrm{NRS}$ in $\mathrm{BGJ}_{5}$.

HISTOLOGY

Sample explants were fixed for histological study as $\frac{\widehat{\sigma}}{\vec{\phi}}$ described by Fell and Barratt (1973). Sections were stained with toluidine blue, Harris's haematoxylin and क chromotrope or celestine blue, Carrazzi's haematoxylin, $\overrightarrow{0}$ and van Gieson's stain.

BIOCHEMICAL ANALYSIS

Proteoglycan was measured by precipitation with alcian blue dye (modified from Whiteman, 1973). The blue 2 complex, after being washed and redissolved in a dis- $\omega$ sociating agent $(40 \%$ Manoxol IB or $2 \%$ sodium dodecyl sulphate, in $50 \mathrm{mmol} / \mathrm{l}$ acetate buffer, $\mathrm{pH} 5.8$ ) was esti- $\dot{\omega}$ mated quantitatively by spectrophotometry at $620 \mathrm{~nm}$. The standard used for comparison was chondroitino sulphate (ex-shark's fin, Koch-Light laboratories). Nine samples containing $10 \mu \mathrm{g}$ chondroitin sulphate gave $a_{\supset}$ mean $\mathrm{E}_{620}$ of 0.60 with a standard error of the mean of 0.01 . A linear relationship was obtained between 0.5 and $\frac{C}{C}$ $15 \mu \mathrm{g}$ chondroitin sulphate per $200 \mu \mathrm{l}$ assay.

Uronic acid was measured by the carbazole method $\overrightarrow{0}$ (Bitter and Muir, 1962). Glucuronolactone was used aş standard.

Collagen was estimated as hydroxyproline after a hydrolysis of the samples at $105^{\circ} \mathrm{C}$ for $24 \mathrm{~h}$ (Woessne 1961 ; modified by Burleigh, Barrett, and Lazarus, 1974). The serum component CIq, present in the medium, contains hydroxyproline, hence blank values had to be $\frac{}{\Phi}$ subtracted from the measurements obtained for the medium in which explants had been cultured.

These assays were made directly on the medium in 3 which the explants had been cultured, and on the explants at the end of each experiment, after solubilization with papain and suitable dilution of the resulting extract. (1) Papain, when tested on known standards, was found not to affect the reaction with alcian blue. The isolated cartilage explants were in the range of $1-4 \mathrm{mg}$, and the explants with invasion zone were in the range $5-16 \mathrm{mg}$, dry weight. The concentrations of proteoglycan and hydroxy-3 proline were expressed as $\mu \mathrm{g} / \mathrm{mg}$ final dry weight. From this and the cumulative figures for the medium, the total percentage release from the cartilage could be calculated as $\frac{D}{2}$ a ratio of product in the medium divided by total product in medium plus cartilage.

\section{Results}

EFFECT OF RETINOL

\section{Depletion of matrix}

Isolated cartilage, devoid of marrow, when grown inco R + NRS had lost most of its proteoglycan (CPG) by the 10th day (Fig. 2); when the explants were ex-? amined after 16 days in culture, less than $20 \%$ of their original content remained in the tissue, as compared $\frac{0}{\mathbb{Q}}$

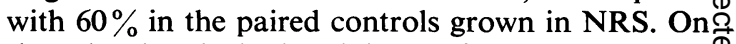
the other hand, the breakdown of collagen, as indi-尺 


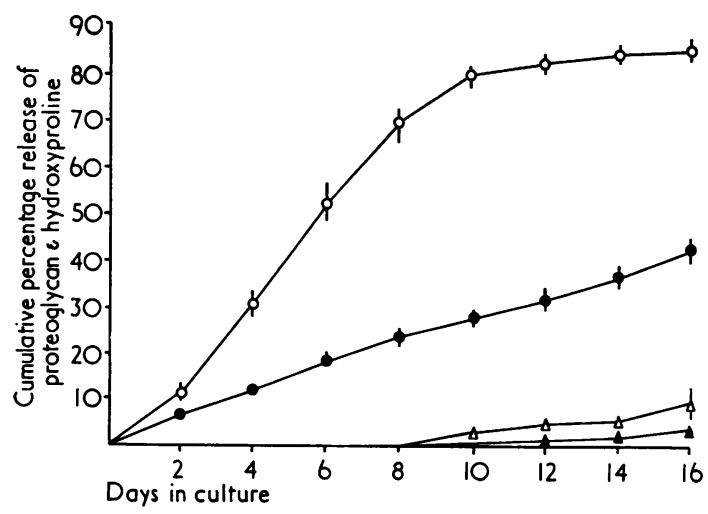

FIG. 2 Cumulative percentage release of proteoglycan (CPG) and hydroxyproline from isolated articular cartilage during 16 days' cultivation in $R+N R S .8$ cultures in each group. Vertical bars represent standard error of the mean. $O$, release of $C P G$ in $R+N R S ; \triangle$, release of hydroxyproline in $R+N R S ; \bullet$, release of $C P G$ in NRS; $\triangle$, release of hydroxyproline in NRS

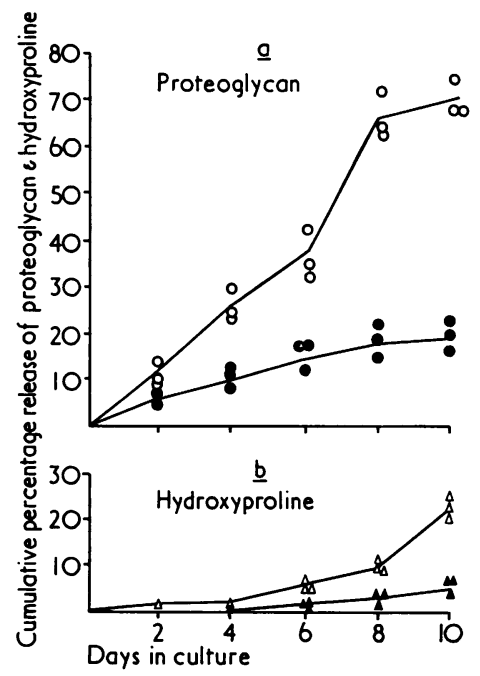

FIG. 3 Cumulative percentage release of CPG (a) and hydroxyproline (b) from cartilage + invasion zone during 10 days' cultivation in $R+N P S ; 3$ cultures in each group. $O$, release of $C P G$ in $R+N P S ; \triangle$, release of hydroxyproline in $R+N P S ; 0$, release of $C P G$ in NPS; $\triangle$, release of hydroxyproline in NPS

cated by the release of hydroxyproline, was slight in both the treated and control explants (Figs 2 and $4 b$ ). The release of CPG was significantly greater than that of the controls by the 2nd day in R + NRS, whereas a significant release of hydroxyproline did not occur until at least the 12th day (Fig. 2).

Cartilage + invasion zone (i.e. cartilage cut to include some invading marrow, see Fig. 1) liberated almost the same amount of CPG in $\mathbf{1 0}$ days in response to added retinol (Fig. $3 a$ ) as the isolated cartilage (Fig. 2). In the breakdown of collagen, however, there was a well-marked difference between the two types of explants; thus cartilage + invasion zone (Fig. $3 b$ ) responded to added retinol by an increased release of hydroxyproline by 10 days; no such change was observed with isolated cartilage (see above Fig. 2). Comparison of the results shown in Figs 2 and 3 is open to the criticism that tissue from different pigs was used. To overcome this difficulty, in one experiment, the breakdown of matrix in cartilage with invasion zone was contrasted with that in isolated cartilage from the same animals (Fig. $4 a, b$ ); essentially the same results were obtained. It will be seen from Fig. 3 that whereas the release of proteoglycan from explants with the invasion zone began during the first 2 days of exposure to retinol, there was a lag of several days before a detectable amount of hydroxyproline was liberated.

Most, but not all, normal rabbit sera contain an inhibitor of collagenase, $\alpha_{2}$-macroglobulin, which must have been present in the pooled sera used for making NRS in the previous experiments. At the suggestion of Dr. J. J. Reynolds an experiment was therefore performed to see whether the absence of this inhibitor would diminish the time-lag observed before hydroxyproline was released from cartilage + invasion zone exposed to retinol. The rabbit serum $\left(\mathrm{NRS}^{1}\right)$ for the media was obtained from a rabbit

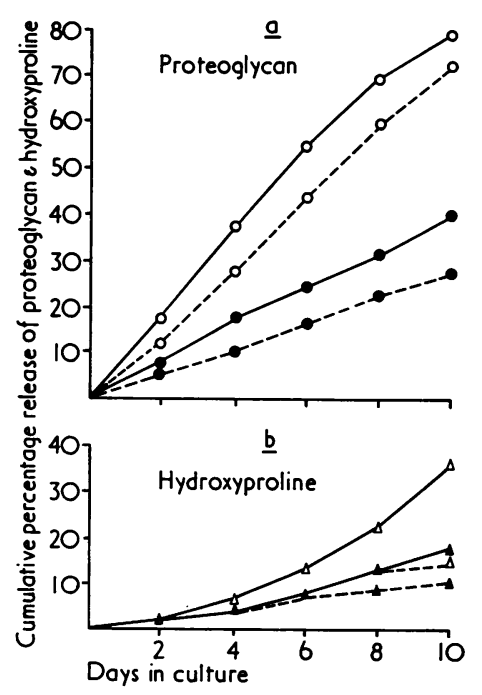

FIG. 4 Direct comparison of the effects of retinol on the release of CPG (a) and hydroxyproline (b) from cartilage with and without the invasion zone. Explants were cultured for 10 days. Each point is the mean of three. $O$, release of $C P G$ in $R+N R S ; 0$, release of $C P G$ in $N R S ; \triangle$, release of hydroxyproline in $R+N R S ; \Delta$, release of hydroxyproline in NRS; — cartilage with invasion zone; - - - isolated cartilage 


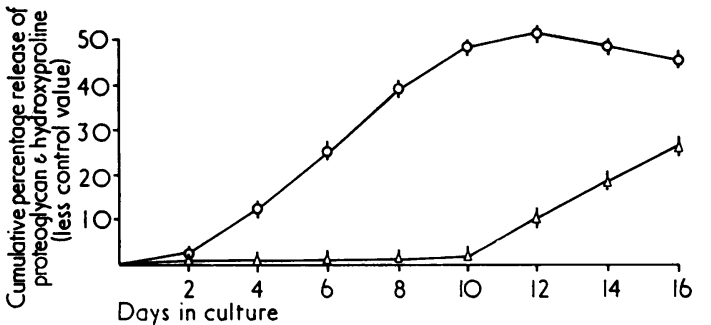

Doys in culture
FIG. 5 Cumulative percentage release of CPG and hydroxyproline from cartilage with invasion zone during 16 days' cultivation in medium containing added retinol but $\mathrm{D}$ lacking the collagenase inhibitor normally present in rabbit serum; 3 cultures in each group. Vertical bars $\vec{\Rightarrow}$ represent standard error of the mean. $\bigcirc$, release of $C P G \stackrel{\oplus}{\oplus}$ in $R+N R S^{\prime}$ as increment over control value; $\triangle$ release $\overline{0}$ of hydroxyproline in $R+N R S^{\prime}$ as increment over control $\frac{}{\bar{\sigma}}$ value

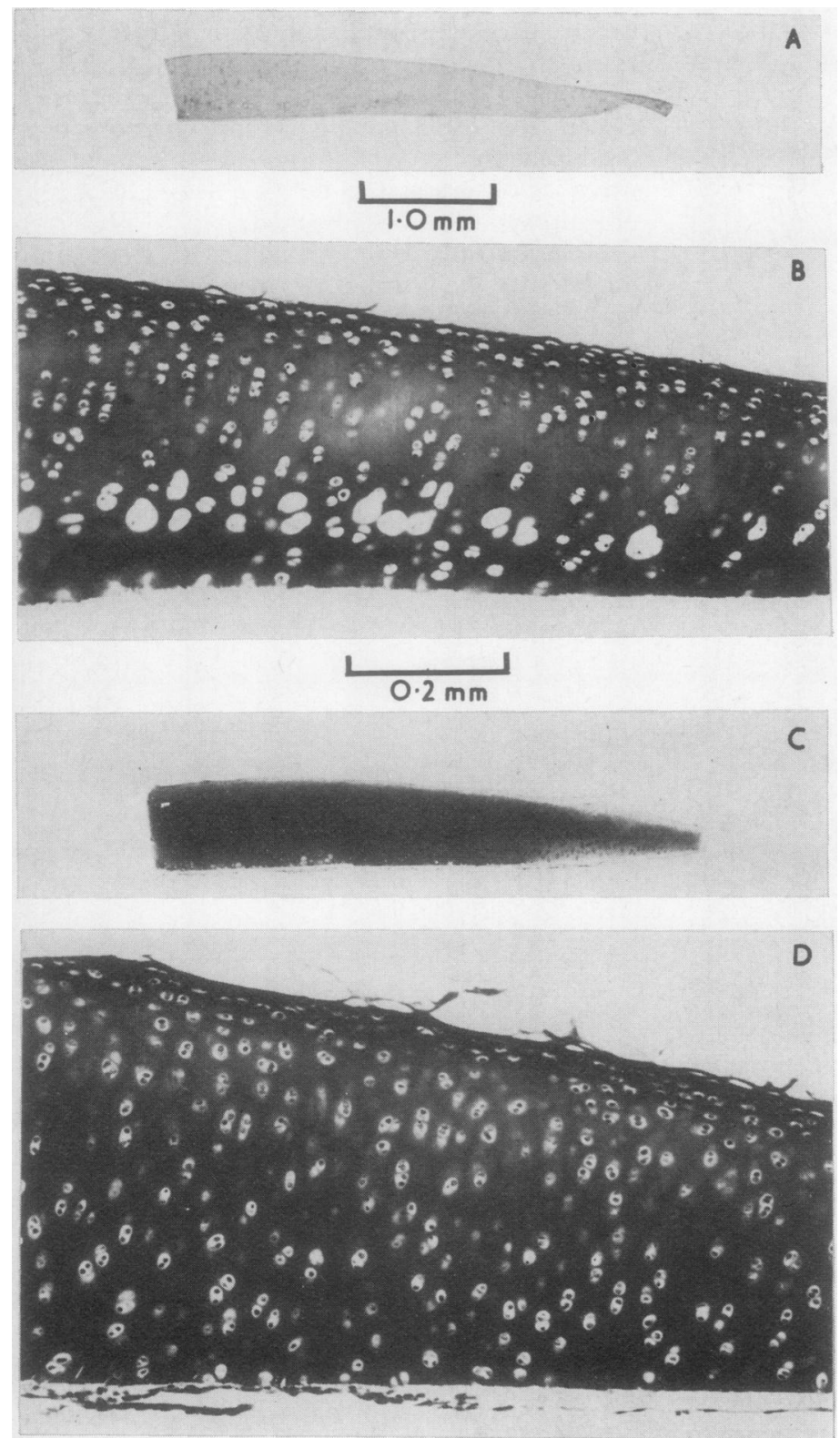

FIG. 6 Paired explants of isolated cartilage cultivated for 16 days; from the same set of cultures as for Fig. 2. (A) Explant cultivated in $R+N R S$, section $\overrightarrow{0}$ stained with toluidine blue; the matrix $B$ nonmetachromatic, indicating severe log of proteoglycan. (B) Same explant, sectiom stained with celestine blue, haematoxylit and van Gieson's stain; the matrix stains as brightly as in the control shown in $D$, confirming that most of the collagen $\stackrel{\circ}{\mathbb{D}}$ has been retained. Note the many round $\Omega$ holes in the matrix, which contain free $\overrightarrow{\overrightarrow{0}}$ chondrocytes and indicate a local re- 3 sorption of collagen. $(C)$ Control cultivated in NRS, section stained with toluidine blue. The matrix is intensely metachromatic. (D) Same explant, section stained with celestine blue, haematoxylin, and van Gieson's stain. The matrix was 3 . stained a brilliant red owing to its high collagen content. Magnification is the same for each figure 
known to lack the inhibitor; 3 pairs of matched explants were cultivated in NRS ${ }^{1}$ for 2 days to remove endogenous inhibitor and then in $R+N^{1} S^{1}$ and (Controls) $\mathrm{NRS}^{1}$ for a further 14 days. The initiation of hydroxyproline release in response to retinol at 10-12 days (8-10 with retinol) was no sooner, nor was the amount released any greater, than in normal medium. (Compare Fig. 5 with Figs $3 b$ and $4 b$.)

Histological examination of sample explants confirmed the results of the biochemical analysis. After 16 days' exposure of isolated cartilage to R + NRS (Fig. $6 A)$ metachromasia had virtually disappeared from the matrix, a row of round holes (Fig. $6 B$ ) containing free dead and living chondrocytes was present a short distance above the cut surface, and just above this surface many of the cells were dead. Most of the paired controls (Fig. 6C) stained almost normally with toluidine blue; in one experiment, in which the explants were too small, there was a substantial loss of metachromasia from some controls, though much less than in the corresponding retinol-treated cartilage. Sections of the same explants stained with celestine blue, haematoxylin, and van Gieson's stain (Fig. $6 B, D$ ) showed no difference in staining reaction between retinol-treated and control cartilage, and in both the van Gieson's stain coloured the matrix a brilliant red. This indicated that although proteoglycan had been lost from the cartilage grown in $\mathrm{R}+\mathrm{NRS}$, the collagen remained almost intact, as indicated also by the biochemical findings.

When the invasion zone was present, the effect of the vitamin was usually obvious in the living explants by the 10th day, and became increasingly conspicuous during further cultivation. As compared with that of paired controls, the articular cartilage became flatter, smaller, and more translucent so that the underlying calcified tissue became visible as if through glass.
Sections of explants fixed after 10 days in $\mathrm{R}+\mathrm{NRS}$ (Fig. 7A) when stained with toluidine blue showed extensive loss of metachromasia in contrast to the intensely metachromatic matrix of the corresponding controls (Fig. 7B) in NRS (see Fell and Barratt, 1973). A well-marked difference between the two sets of explants was also seen in preparations stained with celestine blue, haematoxylin, and van Gieson's stain. In the retinol-treated specimens, in those areas where the cartilage was most severely affected and the chondrocytes had assumed an irregular, fibroblastic form (see Barratt, 1973), the matrix stained very weakly with van Gieson's stain, implying that collagen had been lost in addition to proteoglycan. There was not an accurate correspondence between disappearance of metachromasia and lack of coloration with van Gieson's stain, and the matrix of less affected regions, though no longer metachromatic, stained normally with van Gieson's stain; this observation supported the biochemical finding that loss of proteoglycan precedes that of collagen.

\section{EFFECT OF AS + $\mathbf{C}^{\prime}$ \\ Depletion of matrix}

Biochemical analysis of isolated cartilage in culture (Fig. 8) showed no significant difference in the release of CPG between the treated and control explants, and there was virtually no loss of hydroxyproline in either medium. On the other hand, cartilage + invasion zone (Fig. 9) released much more of both CPG and hydroxyproline in AS $+\mathrm{C}^{\prime}$ than in NRS. The loss of both these components at comparable times was less in AS + $C^{\prime}$ than in R + NRS (cf. Figs 3 and 4). Whereas release of CPG began almost immediately after explantation, that of hydroxyproline was not significantly greater than in the control until the 8th day.

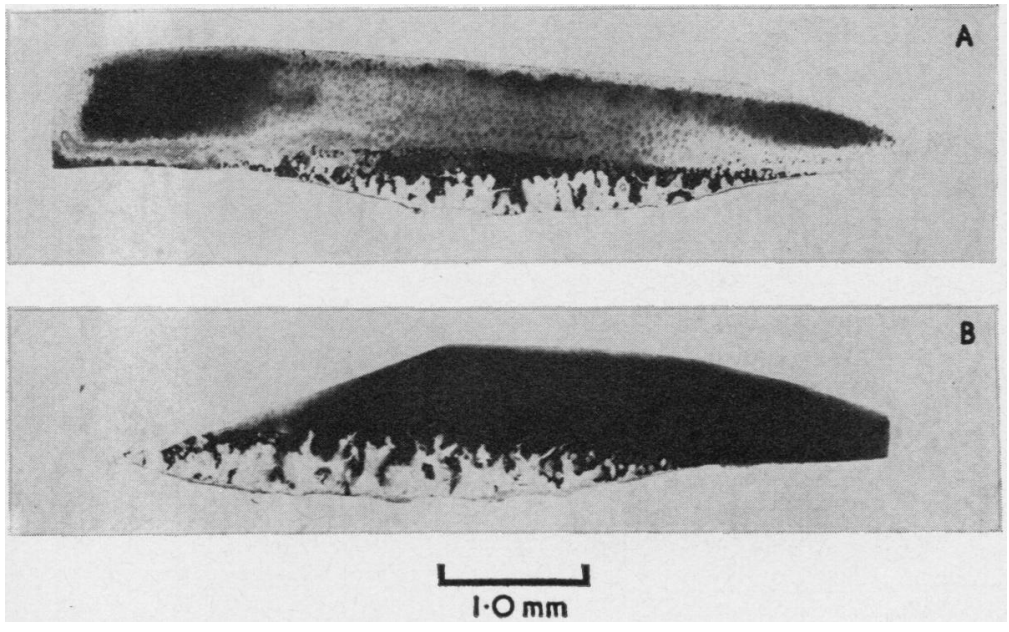

FIG. 7 Explants of cartilage with the invasion zone, cultivated for 10 days; from the same set of cultures as for Fig. 3. Sections stained with toluidine blue. (A) Explant cultivated in $R+N P S$; metachromasia is greatly reduced in the matrix of the noncalcified cartilage. (B) Control cultivated in NPS. All the cartilage matrix is intensely metachromatic 
The structure of sample explants fixed for histological examination was similar to that described by Fell and Barratt (1973). The matrix of isolated cartilage (Fig. 10A) cultured in $\mathrm{AS}+\mathrm{C}^{\prime}$ stained as intensely with both toluidine blue and van Gieson's

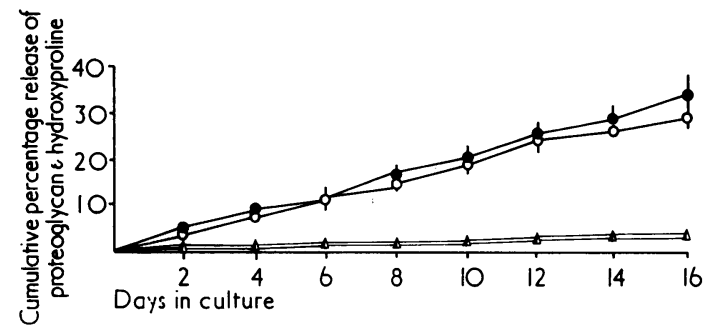

FIG. 8 Release of CPG and hydroxyproline from isolated cartilage during 16 days' cultivation in $A S+C^{\prime} ; 8$ cultures in each group. $\bigcirc$, release of $C P G$ in $A S+C^{\prime} ; \bullet$, release of $C P G$ in NRS; $\triangle$, release of hydroxyproline in NRS; $\triangle$, release of hydroxyproline in $A S+C^{\prime}$

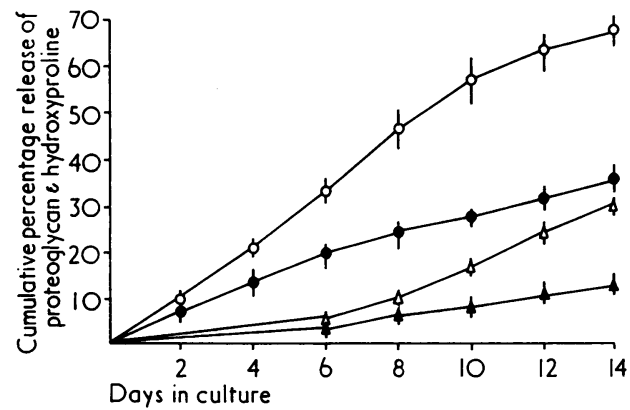

FIG. 9 Release of CPG and hydroxyproline from cartilage with invasion zone, during 14 days' cultivation in $A S+C^{\prime}$; 8 cultures in each group. $\bigcirc$, release of $C P G$ in $A S+C^{\prime}$; $\bullet$, release of $C P G$ in NRS; $\triangle$, release of hydroxyproline in $A S+C^{\prime} ; \Delta$, release of hydroxyproline in NRS. Vertical bars represent standard error of the mean stain as that of the controls in NRS; the controls, however, unlike the explants in AS + $\mathbf{C}^{\prime}$, all formed nodules of new cartilage beneath the cut surface.

On the other hand, the uncalcified matrix of cartilage + invasion zone cultured in $\mathrm{AS}+\mathrm{C}^{\prime}$ (Fig. $11 \mathrm{~A}$ ) showed extensive and sometimes complete losso of metachromasia (indicating depletion of proteo-흘 glycan), but a diminished staining reaction with van Gieson's stain (implying breakdown of collagen (Fig. $\stackrel{\mathbb{Q}}{\complement}$ $11 B)$ ) appeared only in the most severely degraded matrix, thus confirming that the release of proteo- glycan precedes that of collagen. The articular. cartilage of the controls (Fig. 12A) was much larger $\vec{\omega}$ and intensely metachromatic, apart from small colourless patches just above the invasion zone and near the cut surfaces; it stained well with van Gieson's $\frac{\varrho}{\omega}$ stain (Fig. 12B).

\section{SYNTHESIS OF COMPONENTS OF MATRIX}

In the above experiments the degradation of matrix is shown as a percentage release, an expression whose validity depends on the assumption that the total content of the system remains unchanged. Hence, it was necessary to know whether the measurement of increased release of CPG and collagen from cartilage with invasion zone was influenced by increased syn- $\vec{\theta}$ thesis in the explants treated with retinol or AS $+\mathcal{f}^{\prime}$. as compared with their controls. Should this be ș, the apparent breakdown of the matrix in the expeg mental explants would be greater than the figure shown above if the new material were all released into the medium.

The synthesis of collagen and proteoglycan was estimated by subtracting the total amount of product in the group of zero explants from the total amounts in the corresponding paired explants plus medium at the end of the culture period. The resulting mean differences are given in the Table, together with the standard error of the mean difference in each case. In no experiment was there greater synthesis in the 3 .

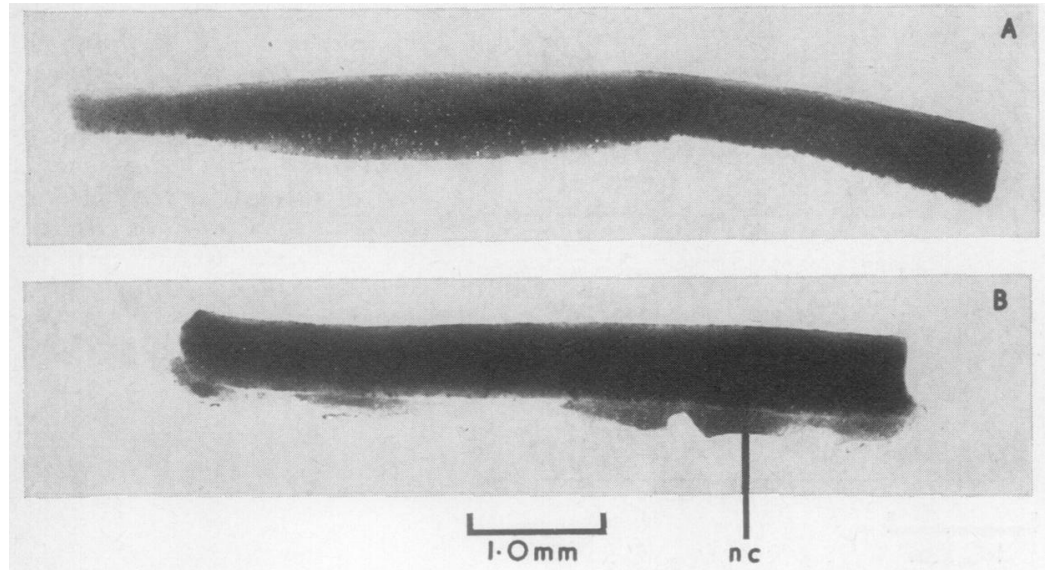

FIG. 10 Explants of isolated cartilage cultivated for 16 days; from the same set of cultures as for Fig. 8. Sections stained with ${ }^{\mathrm{Gr}}$ toluidine blue. (A) Explant cul-N tivated in $A S+C^{\prime}$. (B) Control in NRS. Both specimens are intensely metachromatic, but in $B$ new cartilage ( $n c)$ has been formed beneath the old, $a \bar{D}$ feature not seen in explants? exposed to $A S+C^{\prime}$ 

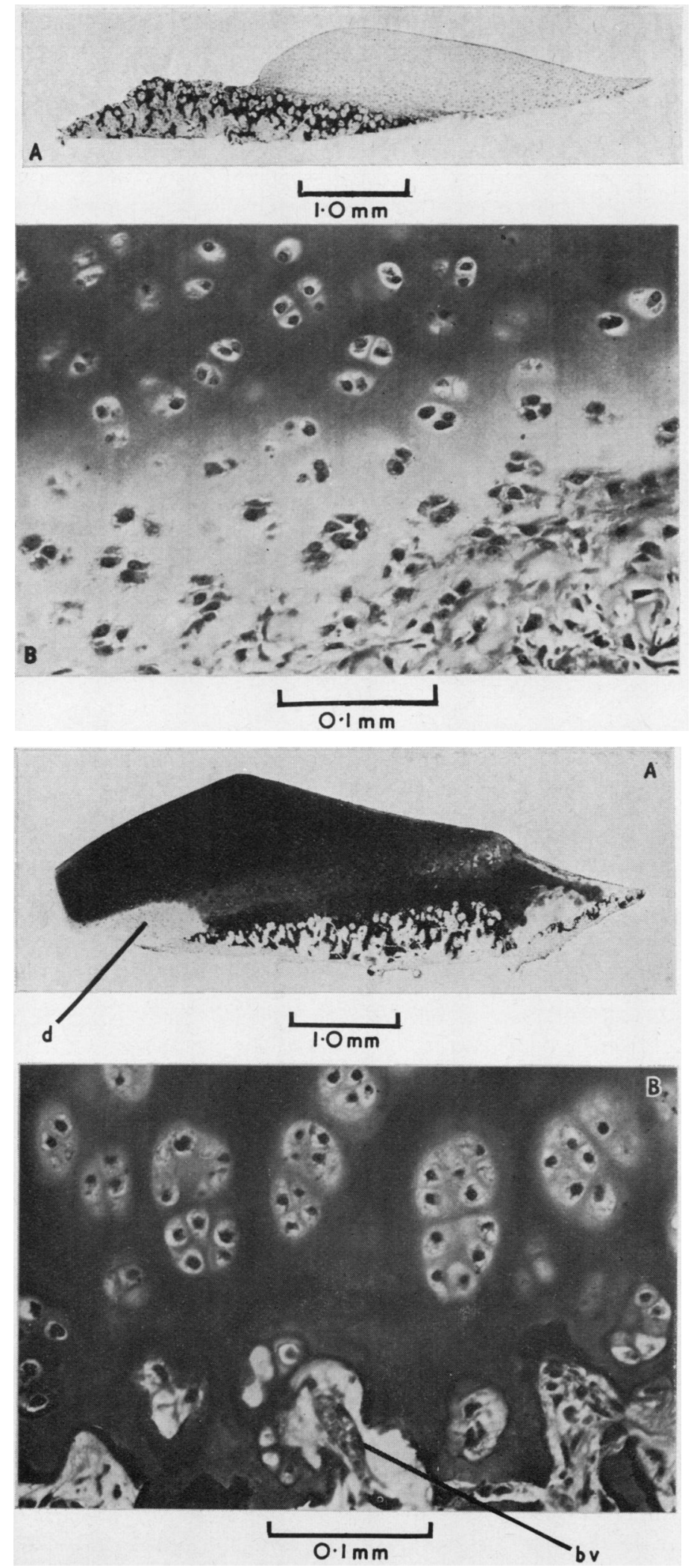

FIG. 11 Explant with the invasion zone, cultivated in $A S+C^{\prime}$ for 14 days; from the same set of cultures as for Fig. 9. (A) Section stained with toluidine blue; note the complete loss of metachromasia throughout the noncalcified cartilage indicating severe depletion of proteoglycan. (B) Same explant, section stained with celestine blue, haematoxylin, and van Gieson's stain, showing part of the region immediately above the invasion zone. In the upper part of the field the matrix stains almost normally with van Gieson's stain, indicating a high collagen content; in the lower portion the chondrocytes have. assumed an irregular, fibroblastic form and the matrix stains faintly, implying. loss of collagen

FIG. 12 Explant with the invasion zone grown in NRS for 14 days; paired control to the specimen shown in Fig. 11. (A) Section stained with toluidine blue; the matrix is intensely metachromatic apart from two small depleted areas $(d)$ just above the invasion zone. (B) Same specimen, section stained with celestine blue, haematoxylin, and van Gieson's stain; comparable region to that shown in Fig. 11B. The invading marrow ( $b v=$ blood vessel) and part of the overlying noncalcified cartilage are seen. The matrix is deeply stained throughout, indicating retention of collagen 
Table Synthesis of matrix components

\begin{tabular}{|c|c|c|c|c|}
\hline Experiment & No. & Treatment & $\begin{array}{l}\text { Increase in hydroxy- } \\
\text { proline (total } \mu \mathrm{g} / \\
\text { culture) over paired } \\
\text { zero explant }\end{array}$ & $\begin{array}{l}\text { Increase in CPG } \\
\text { (total } \mu g \text { chondroitin } \\
\text { sulphate/culture) } \\
\text { over paired zero } \\
\text { explant }\end{array}$ \\
\hline 1 & 10 & $\begin{array}{l}R+N R S \\
\text { NRS }\end{array}$ & $\begin{array}{r}64 \pm 16 \\
140 \pm 22\end{array}$ & $81 \pm 69$ \\
\hline 2 & 10 & $\begin{array}{l}\mathrm{R}+\mathrm{NRS} \\
\mathrm{NRS}\end{array}$ & ${ }^{0} 61 \pm 21$ & $\begin{array}{l}0^{*} \\
120 \pm 54\end{array}$ \\
\hline 3 & 10 & $\begin{array}{l}\mathrm{AS}+\mathrm{C} \\
\mathrm{NRS}\end{array}$ & $\begin{array}{l}0 \\
0\end{array}$ & $\begin{array}{r}48 \pm 36 \\
126 \pm 34\end{array}$ \\
\hline 4 & 9 & $\begin{array}{l}\mathrm{AS}+\mathrm{C} \\
\mathrm{NRS}\end{array}$ & ${ }_{89 \pm 18}$ & $\begin{array}{l}0 \\
115 \pm 45\end{array}$ \\
\hline
\end{tabular}

* See text.

experimental explant than in its control. In the instances marked with an asterisk (zero increase of CPG as measured by alcian blue) the mean of the experimental group was in fact significantly less than that of the 'zero tissue' group. Measurements of the uronic acid in the tissue and medium, when compared with the corresponding estimations of CPG made by means of the alcian blue method, supported the hypothesis that this phenomenon was due to degradation within the tissue of the CPG breakdown products to a level at which the molecular weight was too small to be detected by the alcian blue method, i.e. the figure obtained for CPG in the tissue is too low. This does not conflict with the main conclusion drawn from the above Table, that there is no evidence for an increase in synthesis in the experimental explants as compared with the controls.

\section{Discussion}

The present experiments on the effect of retinol on pig articular cartilage with and without the invasion zone confirmed Barratt's previous finding (Barratt, 1973) that the breakdown of the matrix was greatly enhanced by the presence of the invading marrow; there was one difference, however, between the two sets of results. Using cartilage from younger pigs and a slightly different culture technique, Barratt found much less depletion of metachromatic material in isolated cartilage exposed to the vitamin than was observed in the experiments reported here.

The results described above are compatible with the hypothesis that under the influence of retinol the chondrocytes secreted enzymes, possibly of lysosomal origin, capable of degrading CPG, but that either these cells have little collagenolytic activity or this activity is not readily released by the vitamin. The identity of the chondrocytes' CPGases has not yet been determined, but experiments on rabbit and human articular tissues in which a new assay for
CPGases was used (for review see Dingle, 1974) indicate that cathepsins $\mathrm{D}, \mathrm{B} 1$, and $\mathrm{F}$ are the most likely candidates. There does not appear to be enough neutral proteinase or hyaluronidase in chondrocytes for these enzymes to play a significant role.

Although in the present work the loss of CPG in response to retinol did not depend on the presence of the invasion zone, this was not true of the breakdown of collagen, and much more hydroxyproline was liberated from cartilage that contained invading marrow. This observation would explain why in these as in Barratt's earlier experiments (Barratt, 1973), breakdown of the cartilage was always more advanced in explants with the invasion zone than in those without it.

Complement-sufficient antiserum failed to stimulate release of either CPG or hydroxyproline from isolated cartilage without the invasion zone. When the invasion zone was present, however, both these components of the matrix were liberated, showing that the cells of the invading marrow could secrete both CPGase and collagenase. The liberation of CPG began almost immediately but that of hydroxyproline was not detectable until 4 to 6 days later. There are several possible explanations of this phenomenon. Both collagenase and CPGase may have been released simultaneously, but the effective action of the CPGase may have been much greater than that of the collegenase, either because more was secreted or simply because less degradation of the substrate was needed for the diffusion out of the matrix of the CPG than for that of the collagen. It is possible that collagenase may have been secreted later than CPGase, perhaps influenced by the endocytosis of partially degraded CPG by the invading cells. Reynolds and Werb (unpublished work, 1974) have shown that the endocytosis of indigestible or slowly digestible material greatly increases the production of extracellular collagenase by synovial cells in culture. 
On the other hand CPG may protect collagen from attack by collagenase, so that breakdown of collagen cannot occur until degradation of the CPG has reached a certain stage (Gnädinger, Itoi, Slansky, and Dohlman, 1969; Hook, Brown, Iwanij, and Nakanishi, 1971).

The breakdown of articular cartilage in culture can be achieved in the absence of the invasion zone by combining the explant with a piece of synovial tissue (Fell and Barratt, 1973; Poole and others, 1973). Unpublished analytical experiments by the present authors suggest that the synovium acts like the cells of the invasion zone by secreting CPGase and collagenase into the matrix of the adjacent cartilage. It is probable that a similar enzymatic mechanism op- erates in rheumatoid arthritis during the replacement of the cartilage by ingrowing pannus, though here the process is no doubt complicated by the presence of inflammatory cells and their content of neutral proteinases.

The authors are indebted to the Medical Research Council, the Nuffield Foundation, and the Arthritis and Rheumatism Committee for financial support. H.B.F. wishes to express her gratitude to Professor R. R. A. Coombs, F.R.S., for providing her with laboratory accommodation. The pig material was kindly supplied free of charge by Daisy Meadow Fresh Foods Ltd. Thanks are due to Mr. R. C. Green for his expert technical assistance, and to Mr. Green and Mr. M. F. Applin for the photographs.

\section{References}

BARRATt, M. E. J. (1973) J. Cell Sci. 13, 205 (The role of soft connective tissue in the response of pig articular cartilage in organ culture to excess of retinol)

BitTer, T., AND MUIR, H. M. (1962) Analyt. Biochem., 4, 330 (A modified uronic acid carbazole reaction)

Burleigh, M. C., Barrett, A. J., AND LaZARUS, G. S. (1974) Biochem. J., 137, 387 (Cathepsin B1 : a lysosomal enzyme that degrades native collagen)

DiNGLE, J. T. (1974) 'Matrix degrading enzymes', in 'Stanmore Symposium on Normal and Osteoarthritic Cartilage', Institute of Orthopedics, University of London

Fell, H. B., AND Barratt, M. E. J. (1973) Int. Arch. Allergy, 44, 441 (The role of soft connective tissue in the breakdown of pig articular cartilage cultivated in the presence of complement-sufficient antiserum to pig erythrocytes: I. Histological changes)

—, AND Dingle, J. T. (1969) J. Cell. Sci., 4, 89 (Endocytosis of sugars in embryonic skeletal tissues in organ culture)

Gnädinger, M. C., Itoi, M., Slansky, H. H., AND Dohlman, C. H. (1969) Amer. J. Ophthal., 68, 478 (The role of collagenase in the alkali-burned cornea)

Hook, C. W., Brown, S. I., Iwanis, W., and Nakanishi, I. (1971) Invest. Ophthal., 10, 496 (Characterization and inhibition of corneal collagenase)

Poole, A. R., Barratt, M. E. J., AND Fell, H. B. (1973) Int. Arch. Allergy, 44, 469 (The role of soft connective tissue in the breakdown of pig articular cartilage cultivated in the presence of complement-sufficient antiserum to pig erythrocytes: II. Distribution of immunoglobulin G)

Whiteman, P. (1973) Biochem. J., 131, 343 (The quantitative measurement of Alcian blue-glycosaminologlycan complexes)

WoEsSNer, J. F. (1961) Arch. Biochem., Biophys., 93, 440 (The determination of hydroxyproline in tissue and protein samples containing small proportions of this imino acid) 